\title{
5 ESTUDO DAS PROPRIEDADES PSICOMÉTRICAS DA ESCALA DE BEM-ESTAR PSICOLÓGICO DE 42 ITENS
}

| Rosa Maria Freire ${ }^{1}$; Maria Rui Sousa ${ }^{2}$; Filipe Pereira ${ }^{3}$; Teresa Martins ${ }^{4} \mid$

\section{RESUMO}

CONTEXTO: O bem-estar psicológico é um constructo multidimensional que tem merecido atenção dos investigadores no âmbito da saúde mental positiva, na medida em que se relaciona com o desenvolvimento do potencial humano.

OBJETIVO: Validar, para a população portuguesa, a versão reduzida de 42 itens da Escala de Bem-Estar Psicológico numa amostra de adultos e jovens adultos.

METODOLOGIA: Recorremos à análise fatorial confirmatória e à validade de constructo numa amostra de 252 participantes recrutados a partir de uma instituição de ensino superior, sendo $48 \%$ estudantes e $52 \%$ docentes e não docentes, a maioria do sexo feminino com média de idade de 31,4 anos e uma escolaridade média de 15,5 anos. Os participantes preencheram um questionário através de uma plataforma informática. A Escala de Bem-Estar Psicológico é constituída por seis constructos teóricos medidos numa escala de resposta do tipo likert.

RESULTADOS: A análise fatorial confirmatória mostra índices de ajustamento sofrível tendo, no entanto, melhorado com a eliminação de dois itens. As dimensões da escala apresentaram valores de coeficiente alfa de Cronbach com variação entre 0,71 e 0,82 e uma matriz de correlação que varia entre 0,31 a 0,73 , com valores significativos ao nível de 0,01 . Apenas a Autonomia se correlacionou com a dimensão Saúde Mental.

CONCLUSÃO: A escala em estudo tem mostrado adequação para avaliar o bem-estar psicológico. Esta versão mostra ser discriminativa para a idade, género, escolaridade e condição de saúde mental e uma boa consistência interna. A estrutura fatorial da escala não mostra ser tão clara quanto o quadro teórico sugerido, requerendo novas investigações.

\section{PALAVRAS-CHAVE: Estudos de validação; Saúde mental; Avaliação de programas e instrumentos de pesquisa}

\section{RESUMEN}

\section{"Estudio de las propiedades psicométricas de la Escala de Bien- estar Psicológico de 42 ítems"}

CONTEXTO: El bienestar psicológico es un constructo multidimensional que ha merecido la atención de los investigadores en el ámbito de la salud mental positiva en la medida en que se refiere al desarrollo del potencial humano.

OBJETIVO: Validar para la populación portuguesa la versión reducida de 42 ítems de la Escala de Bienestar Psicológico.

METODOLOGIA: Aprobemos al análisis factorial confirmatorio ya la validez de constructo en una muestra de 252 participantes reclutados a partir de una institución de enseñanza superior, siendo $48 \%$ estudiantes y $52 \%$ docentes y no docentes, la mayoría del sexo femenino con media de edad de 31, 4 años y una escolaridad promedio de 15,5 años. Los participantes llenaron un cuestionario a través de una plataforma informática. La Escala de Bienestar Psicológico está constituida por seis constructos teóricos medidos en una escala de respuesta del tipo likert.

RESULTADOS: El análisis factorial confirmatorio muestra índices de ajuste sufrido, sin embargo, mejorado con la eliminación de dos ítems. Las dimensiones de la escala presentaron valores de coeficiente alfa de Cronbach con variación entre 0,71 y 0,82 y una matriz de correlación que varía entre 0,31 a 0,73 , con valores significativos al nivel de 0,01 . Sólo la Autonomía se correlacionó con la dimensión Salud Mental.

CONCLUSIÓN: La escala en estudio ha mostrado adecuación para evaluar el bienestar psicológico. La versión en estudio muestra ser discriminatoria para la edad, género, escolaridad y condición de salud mental y una buena consistencia interna. La estructura factorial de la escala no muestra ser tan clara como el cuadro teórico sugerido, requiriendo nuevas investigaciones.

DESCRIPTORES: Estudios de validación; Salud mental; Evaluación de programas e instrumentos de investigación

\section{ABSTRACT \\ "Psychometric properties of the Psychological Well-Being Scale of 42 items"}

BACKGROUND: The psychological well-being is a multidimensional construct that has deserved the attention of researchers in the field of positive mental health as it relates to the development of human potential. AIM: To validate for Portuguese the reduced version of 42 items of the Psychological Well-Being Scale.

METHODS: We used confirmatory factor analysis and construct validity in a sample of 252 participants recruited from a higher education institution, of which $48 \%$ were students and $52 \%$ were teachers and nonteachers, the majority are female, with a mean age of 31,4 years old and an average schooling of 15.5 years. Participants completed a questionnaire through a computer platform. The Psychological Welfare Scale consists of six theoretical constructs measured on a likert-type response scale.

RESULTS: Confirmatory factorial analysis showed satisfactory adjustment indexes, however, improved with the elimination of two items. The scale dimensions presented values of Cronbach's alpha coefficient ranging from 0.71 to 0.82 and a correlation matrix ranging from 0.31 to 0.73 , with significant values at the 0.01 level. Autonomy alone correlated with the Mental Health dimension.

CONCLUSION: The scale in study has shown adequacy to evaluate the psychological well-being. The study version shows to be discriminative for age, gender, schooling and mental health condition and a good internal consistency. The factorial structure of the scale does not show to be as clear as the theoretical framework suggested, requiring further investigations.

\section{KEYWORDS: Validation studies; Mental health; Evaluation of research programs and tools}

Submetido em 30-09-2018

Aceite em 24-11-2018

1 Doutoranda; Mestre; Professora Adjunta na Escola Superior de Enfermagem do Porto, 4200-072 Porto, Portugal, rosafreire@esenf.pt

2 Doutora; Investigadora Integrada no CINTESIS - Centro de Investigação em Tecnologias e Serviços de Saúde, grupo de investigação "NursID - Investigação e Desenvolvimento em Enfermagem"; Professora Adjunta na Escola Superior de Enfermagem do Porto, 4200-072 Porto, Portugal, mariarui@esenf.pt

3 Doutor; Professor Coordenador na Escola Superior de Enfermagem do Porto, 4200-072 Porto, Portugal, filipereira@esenf.pt

4 Doutora; Investigadora Integrada no CINTESIS - Centro de Investigação em Tecnologias e Serviços de Saúde, grupo de investigação "NursID - Investigação e Desenvolvimento em Enfermagem"; Professora Coordenadora na Escola Superior de Enfermagem do Porto, 4200-072 Porto, Portugal, teresam@esenf.pt

Citação: Freire, R. M., Sousa, M., Pereira, F., \& Martins, T. (2019). Estudo das propriedades psicométricas da Escala de Bem-Estar Psicológico de 42 itens. Revista Portuguesa de Enfermagem de Saúde Mental (21), 31-xx 


\section{INTRODUÇÃO}

A definição de bem-estar tem evoluído ao longo dos tempos. A compreensão do bem-estar como "ausência de doença" foi sendo substituída por outros termos mais específicos que requerem distinção. $\mathrm{O}$ bem-estar subjetivo relaciona-se com duas dimensões: uma associada a satisfação com a vida, de cariz cognitivo, e outra que balanceia afetos positivos e negativos, de natureza afetiva (Ryff, 2014). O bem-estar subjetivo é imbuído em valores, necessidades, crenças e espectativas pessoais (Novo, 2005), tidos em conta quando se avalia o prazer e a felicidade. Esta perspetiva hedónica do bem-estar é considerada como bem-estar subjetivo. $\mathrm{O}$ bem-estar psicológico, com raízes na era aristotélica, aposta no alcance da felicidade com o desenvolvimento do primado da realização pessoal e é considerado como bem-estar eudaimónico (Malla, 2013).

$\mathrm{O}$ interesse pelo estudo do bem-estar psicológico emergiu na década de 80 e teve o seu auge com um trabalho desenvolvido nos Estados Unidos (Ryff, 1989) que tem por base a perspetiva eudaimónica do bem-estar, ou seja, com enfoque no desenvolvimento do potencial humano. Neste âmbito, a autora relaciona a saúde mental positiva com o bem-estar psicológico, que inclui a avaliação que o indivíduo faz da sua vida no que se refere a sentimentos de satisfação e felicidade, condições da vida que detém na área relacional e social e realizações pessoais anteriores e expectativas futuras (Novo, 2003). $\mathrm{O}$ modelo de bem-estar psicológico inclui contributos teóricos de vários estudiosos da saúde mental positiva, nomeadamente de Maslow, Roggers, Jahoda, Erikson e Neugarten (Ryff, 1989). O modelo de Ryff é multifacetado e relaciona constructos teóricos como Autonomia, Aceitação de Si, Domínio do Meio, Objetivos de Vida, Relações Positivas com os Outros e Crescimento Pessoal.

A Autonomia avalia a autodeterminação, a resistência a pressões sociais e padrões pessoais de resposta. A Aceitação de Si determina a atitude em relação a si próprio, a aceitação de boas e más qualidades e da vida passada. $\mathrm{O}$ Domínio do Meio caracteriza a competência para gerir o meio ambiente de forma a criar contextos adequados às necessidades e valores pessoais.

Os Objetivos de Vida avaliam a posse de propósitos e metas que orientam e dão significado à vida. As Relações Positivas com os Outros qualificam os relacionamentos interpessoais. O Crescimento Pessoal considera o permanente desenvolvimento do potencial humano com a finalidade da sua maximização.
Estes constructos são espelhados em seis escalas independentes e autónomas que, quando aplicadas conjuntamente, constituem um inventário único e enformam a Escala de Bem-estar Psicológico (EBEP). Inicialmente composta por 120 itens (20 por cada escala), foi progressivamente alterada e tomando dimensões mais reduzidas. Ryff propôs uma versão de 84 itens (14 itens por dimensão), sendo esta versão a mais utilizada pela autora e seus colaboradores em vários estudos (Novo, 2005). Existem versões com 54 itens (van Dierendonck, 2005), 42 itens (Boylan \& Ryff, 2015) e 18 itens (Ryff \& Keyes, 1995). Esta última versão é apenas recomendada para estudos de grandes dimensões, que requeiram brevidade e economia (Ryff \& Keyes, 1995). Segundo Ryff (2014) a EBEP encontra-se traduzida para mais de 30 idiomas diferentes e conta com mais de 350 publicações, em mais de 150 revistas científicas.

A adaptação da EBEP para a população portuguesa foi efetuada com a versão de 84 itens (Novo, 2003, 2005). Fernandes, Vasconcelos-Raposo e Teixeira (2010) e Fernandes, Vasconcelos-Raposo, Bertelli e Almeida (2011) estudaram as características psicométricas de uma versão portuguesa com 30 e com 18 itens (Fernandes et al., 2010; Fernandes et al., 2011). Porém, as versões mais curtas mostram-se pouco específicas e pouco robustas (Ryff, 2014; van Dierendonck, 2005). Com base nos resultados do projeto MIDUS (Morozink, Friedman, Coe, \& Ryff, 2010) a autora sugere que o uso de uma versão reduzida seja efetuado com 42 itens. Esta medida utilizada em estudos recentes (Abbott, Ploubidis, Huppert, Kuh, \& Courdace, 2010; Boylan \& Ryff, 2015; Villarosa \& Ganotice, 2018) mostra boas propriedades psicométricas. Porém, a sua inexistência, no contexto português, determina a necessidade de estudar as características psicométricas da versão de Boylan e Ryff (2015).

\section{METODOLOGIA}

Desenvolveu-se um estudo metodológico do tipo transversal. Não se efetuou a tradução e adaptação linguística da escala em estudo, uma vez que Novo (2003) apresentou este processo para a versão de 84 itens. Assim, os itens em análise foram selecionados a partir do seu trabalho.

\section{Participantes}

Os participantes em estudo foram selecionados segundo uma amostra de conveniência, tendo por base a comunidade educativa de uma escola superior de enfermagem do norte de Portugal (docentes, não docentes e estudantes). 
Os critérios de inclusão aplicados foram: ter idade igual ou superior a 18 anos, ter um mail da instituição atribuído e participar voluntariamente no estudo.

De entre os 1400 contactos elegíveis, 85,4\% (1195) eram estudantes, $11,6 \%$ (163) eram docentes internos ou externos e 3\% (42) pertencia ao grupo não docente. Responderam 544 participantes, tendo sido eliminados 292 questionários por não incluírem informação sociodemográfica e/ou por ter mais de oito itens da EBEP com valores omissos.

A amostra final ficou constituída por 252 participantes, sendo 48\% (121) estudantes e 52\% (131) docentes e não docentes. Os participantes tinham uma média de idade de 31,38 anos $(\mathrm{DP}=12,88)$, tendo variado entre os $18 \mathrm{e}$ os 68 anos. Cento e vinte e quatro participantes $(49,2 \%)$ tinham idades inferiores ou igual a 25 anos, 13,5\% (34) situava-se entre 26 e 32 anos e os restantes 37,3\% (94) tinha 33 ou mais anos. A grande maioria dos participantes $(81 \%)$ era do sexo feminino. Quanto ao estado civil, $63,1 \%$ (159) eram solteiros e 32,9\% (83) casados. Apresentavam uma escolaridade média de 15,5 anos $(\mathrm{DP}=3,27 ; \mathrm{Med}=15)$, variando entre os 4 e os 24 anos de formação académica.

\section{Material}

O presente estudo baseou-se na versão da EBEP de 42 itens, proposta por Boylan e Ryff (2015).

A EBEP é um instrumento de autopreenchimento constituído por afirmações descritivas, que na sua apresentação surge com a mistura dos itens das diferentes dimensões. Tendo em consideração a dimensão a que pertencem, as afirmações são apresentadas de forma alternada e referem-se a aspetos favoráveis ou desfavoráveis ao bem-estar psicológico, medidos numa escala de resposta do tipo likert de seis pontos (de discordo completamente a concordo completamente). Cada dimensão tem metade dos itens enunciados de forma positiva e outra metade de forma negativa, para controlo de atitudes de resposta relacionadas com a tendência para a aquiescência. Para o cálculo da pontuação de cada dimensão procede-se ao somatório das pontuações dos itens que a compõe, antecedido da inversão dos itens apresentados de forma negativa, tal como sugerem os autores. Cada dimensão oscila entre 7 e 42 pontos; uma pontuação mais elevada sugere maior perceção de bemestar psicológico nesse domínio.

Para além da EBEP utilizou-se um questionário sociodemográfico (sexo, idade, escolaridade e estado civil) e a versão portuguesa do SF36 que avalia a qualidade de vida (Ferreira, 2000).
Esta medida contempla 36 itens organizados em oito subescalas: Capacidade Funcional; Desempenho Físico; Desempenho Emocional; Dor Corporal; Saúde Mental; Funcionamento Social; Vitalidade e Estado Geral de Saúde. O SF36 contempla ainda um item que quantifica as alterações de saúde durante o último ano. Não existe um padrão único de mensuração dos itens desta escala, havendo possibilidades de resposta dicotómica, ou de escalas ordinais que variam de 1 a 3 , de 1 a 5 ou de 1 a 6. Seguindo as indicações dos autores cada dimensão, após recodificação de itens assinalados varia entre $0 \mathrm{e}$ 100; valores mais elevados correspondem a uma melhor qualidade de vida.

\section{Procedimentos}

A cada participante foi enviada uma mensagem eletrónica, a solicitar a participação no estudo e onde constava o link para aceder ao questionário. Para facilitar a tomada de decisão foi disponibilizada informação sobre a finalidade e os objetivos do estudo, o tempo previsto para o seu preenchimento (15 minutos) e assegurada a confidencialidade e o anonimato do participante. $\mathrm{O}$ questionário foi elaborado com recurso ao programa Qualtrics. O estudo teve apreciação favorável e anuência do concelho diretivo da instituição académica envolvida. Foram obtidas autorizações dos autores da versão original e da versão traduzida para português da EBEP e dos autores do SF36.

Para o tratamento estatístico dos dados utilizou-se o programa IBM SPSS ${ }^{\circledast}$ versão 24.0 e estatística paramétrica. Numa primeira etapa, os dados foram explorados através de estatística descritiva. Foram utilizadas medidas de correlação, nomeadamente a correlação de Pearson, para estudar a força de associação entre variáveis contínuas. Na comparação de médias entre amostras independentes utilizou-se o teste $\mathrm{t}$ de Student, tendo sido previamente verificado os pressupostos para a sua utilização. A consistência interna foi determinada pelo coeficiente alfa de Cronbach. Dado a maioria dos estudos sugerir a estrutura hexagonal da EBEP (Ryff \& Keyes 1995; Ryff \& Singer, 2006; van Dierendonck, 2005), optamos por estudar a validade fatorial através da análise fatorial confirmatória (AFC), com recurso ao AMOS (versão 24, IBM SPSS). A existência de outliers foi avaliada pela distância quadrada de Mahalonobis e a normalidade avaliada pelo coeficiente de simetria e curtose uni e multivariada. Considerou-se como entrada a matriz de covariância, adotando-se o método da máxima verossimilhança de estimação. 
O ajustamento local foi avaliado pelos pesos fatoriais e pela fiabilidade individual dos itens. O ajustamento do modelo teve para além dos índices de modificação as considerações teóricas subjacentes e as recomendações de Kline (2010). $\mathrm{Na}$ avaliação dos modelos foram utilizadas medidas de ajuste que incluíram os índices de aderência Qui-quadrado normalizado ( $\chi 2 / \mathrm{df}$ ), o RMR (root mean square residual), o GFI (goodness of fit index), o CFI (comparative fit index), o índice de parcimónia RMSEA (root mean square error of aproximation) e o ECVI (expected cross validation index). Na presença de menos de oito afirmações da EBEP com valores omissos, estes foram estimados através do método de imputação simples, via um modelo de regressão com estimativas baseadas em máxima verosimilhança. A análise da validade convergente da EBEP do modelo que demonstrou melhor ajustamento ao modelo teórico foi realizada com recurso ao SF36.

\section{RESULTADOS}

Através de uma análise exploratória (tabela 1) verificamos o total de 849 (8\%) valores omissos, tendo o item 1 do Crescimento Pessoal registado a menor percentagem (2\%) e o item 7 do Domínio do Meio a maior (17,9\%). A taxa de não resposta foi superior a $10 \%$ em dez itens. De um modo geral os itens formulados com conteúdos negativos mostraram ter uma maior taxa de não resposta. Verificamos que nas afirmações com conteúdos positivos os participantes posicionaram-se, de um modo geral, nas pontuações mais elevadas de bem-estar, enquanto que nos itens com conteúdos negativos esta tendência não é tão visível. De forma global, a tendência de resposta situou-se acima do ponto médio da escala de medida. Em oito dos itens não se registou qualquer resposta na categoria mais baixa e em sete itens, mais de $40 \%$ das respostas, pontuaram na categoria mais elevada, mostrando um efeito de teto.

Tabela 1 - Estatística Descritiva e Frequências das Categorias de Resposta da Escala do EBEP

\begin{tabular}{|c|c|c|c|c|c|c|c|c|c|c|c|}
\hline \multicolumn{12}{|c|}{ Categorias de resposta } \\
\hline Item & $1(\%)$ & $2(\%)$ & $3(\%)$ & $4(\%)$ & $5(\%)$ & $6(\%)$ & Missing $(\%)$ & $M^{*}$ & $\mathrm{DP}$ & Simetria & Curtose \\
\hline $\mathrm{A} 1+(\mathrm{Q} 5)$ & 0,8 & 3,6 & 6,0 & 26,6 & 34,9 & 22,2 & 6,0 & 4,40 & 1,52 & $-1,46$ & 2,02 \\
\hline $\mathrm{A} 2+(\mathrm{Q} 10)$ & 1,2 & 7,1 & 13,1 & 25,4 & 31,0 & 12,7 & 9,5 & 3,87 & 1,69 & $-0,99$ & 0,27 \\
\hline A3-(Q14) & 3,6 & 15,1 & 29,8 & 10,7 & 17,5 & 12,7 & 10,7 & 3,29 & 1,77 & $-0,21$ & $-0,70$ \\
\hline A4-(Q22) & 0,4 & 7,5 & 18,3 & 17,1 & 25,4 & 16,7 & 14,7 & 3,65 & 1,92 & $-0,70$ & $-0,55$ \\
\hline A5+(Q26) & 0,0 & 2,0 & 4,8 & 33,7 & 34,1 & 17,9 & 7,5 & 4,31 & 1,52 & $-1,57$ & 2,44 \\
\hline A6-(Q29) & 2,4 & 9,1 & 19,4 & 17,9 & 23,0 & 21,4 & 6,7 & 3,94 & 1,72 & $-0,68$ & $-0,24$ \\
\hline $\mathrm{A} 7+(\mathrm{Q} 41)$ & 0,4 & 1,6 & 2,0 & 17,9 & 35,7 & 36,1 & 6,3 & 4,76 & 1,54 & $-1,92$ & 3,48 \\
\hline $\mathrm{DM} 1+(\mathrm{Q} 1)$ & 4,0 & 2,0 & 2,8 & 11,9 & 45,2 & 31,7 & 2,4 & 4,81 & 1,39 & $-1,87$ & 3,41 \\
\hline DM2-(Q6) & 5,2 & 22,2 & 34,1 & 10,3 & 15,5 & 4,8 & 7,9 & 2,99 & 1,51 & $-0,03$ & $-0,34$ \\
\hline DM3-(Q11) & 0,4 & 2,4 & 6,7 & 15,1 & 27,8 & 40,1 & 7,5 & 4,65 & 1,70 & $-1,57$ & 1,82 \\
\hline $\mathrm{DM} 4+(\mathrm{Q} 15)$ & 0,4 & 0,4 & 5,2 & 20,6 & 48,0 & 22,2 & 3,2 & 4,73 & 1,21 & $-2,02$ & 5,63 \\
\hline DM5-(Q18) & 3,6 & 13,9 & 21,8 & 12,7 & 22,2 & 14,3 & 11,5 & 3,44 & 1,85 & $-0,39$ & $-0,82$ \\
\hline DM6-(Q38) & 2,4 & 7,9 & 21,0 & 11,1 & 25,4 & 22,6 & 9,5 & 3,88 & 1,85 & $-0,69$ & $-0,46$ \\
\hline $\mathrm{DM} 7+(\mathrm{Q} 42)$ & 1,6 & 3,2 & 5,6 & 17,5 & 31,7 & 22,6 & 17,9 & 3,89 & 2,10 & $-0,94$ & $-0,53$ \\
\hline CP1-(Q2) & 0,0 & 0,4 & 2,4 & 7,1 & 20,6 & 66,5 & 2,0 & 5,44 & 1,09 & $-3,02$ & 11,16 \\
\hline $\mathrm{CP} 2+(\mathrm{Q} 19)$ & 0,0 & 1,2 & 1,2 & 17,1 & 38,1 & 35,3 & 7,1 & 4,77 & 1,56 & $-2,01$ & 3,74 \\
\hline CP3-(Q23) & 1,6 & 3,6 & 6,3 & 11,1 & 28,2 & 43,7 & 5,6 & 4,75 & 1,65 & $-1,60$ & 1,87 \\
\hline $\mathrm{CP} 4+(\mathrm{Q} 27)$ & 1,6 & 0,4 & 2,4 & 20,6 & 34,5 & 35,3 & 5,2 & 4,77 & 1,48 & $-1,90$ & 3,66 \\
\hline CP5-(Q30) & 2,8 & 6,0 & 21,8 & 17,1 & 21,8 & 18,7 & 11,9 & 3,69 & 1,87 & $-0,64$ & $-0,51$ \\
\hline $\mathrm{CP} 6+(\mathrm{Q} 34)$ & 0,4 & 0,4 & 0,4 & 13,9 & 25,4 & 57,1 & 2,4 & 5,28 & 1,16 & $-2,63$ & 8,77 \\
\hline CP7-(Q39) & 0,8 & 2,8 & 3,6 & 12,7 & 24,6 & 48,0 & 7,5 & 4,79 & 1,73 & $-1,72$ & 2,11 \\
\hline $\mathrm{RP} 1+(\mathrm{Q} 3)$ & 0,0 & 1,2 & 1,6 & 26,2 & 46,8 & 14,7 & 9,5 & 4,34 & 1,59 & $-1,80$ & 2,63 \\
\hline RP2-(Q7) & 1,6 & 3,2 & 10,7 & 11,9 & 26,6 & 34,5 & 11,5 & 4,28 & 1,95 & $-1,13$ & 0,14 \\
\hline RP3-(Q12) & 2,4 & 7,9 & 10,3 & 7,5 & 23,0 & 38,5 & 10,3 & 4,25 & 2,00 & $-0,98$ & $-0,32$ \\
\hline $\mathrm{RP} 4+(\mathrm{Q} 16)$ & 0,8 & 4,8 & 2,8 & 15,5 & 38,1 & 33,7 & 4,4 & 4,73 & 1,48 & $-1,73$ & 2,83 \\
\hline RP5+(Q28) & 0,4 & 1,2 & 1,6 & 24,6 & 38,1 & 23,8 & 10,3 & 4,39 & 1,72 & $-1,62$ & 1,84 \\
\hline
\end{tabular}




\begin{tabular}{|l|c|c|c|c|c|c|c|c|c|c|c|}
\hline RP6-(Q31) & 2,8 & 7,5 & 10,7 & 12,7 & 23,0 & 32,1 & 11,1 & 4,09 & 1,99 & $-0,87$ & $-0,44$ \\
\hline RP7+(Q37) & 0,0 & 0,8 & 1,6 & 18,7 & 37,7 & 36,5 & 4,8 & 4,88 & 1,37 & $-2,16$ & 5,35 \\
\hline AS1+(Q4) & 0,0 & 4,8 & 9,5 & 28,6 & 38,5 & 11,9 & 6,7 & 4,16 & 1,49 & 1,82 & 4,40 \\
\hline AS2+(Q9) & 0,0 & 5,2 & 7,9 & 21,8 & 37,3 & 21,0 & 6,7 & 4,34 & 1,57 & $-1,38$ & 1,58 \\
\hline AS3-(Q13) & 3,6 & 12,3 & 20,6 & 7,1 & 23,0 & 19,4 & 13,9 & 3,50 & 2,00 & $-0,40$ & $-1,02$ \\
\hline AS4+(Q21) & 0,0 & 2,4 & 4,4 & 25,8 & 39,7 & 21,4 & 6,3 & 4,48 & 1,48 & $-1,70$ & 2,96 \\
\hline AS5-(Q33) & 9,1 & 13,9 & 15,9 & 8,7 & 18,7 & 25,0 & 8,7 & 3,63 & 2,00 & $-0,33$ & $-1,17$ \\
\hline AS6-(Q36) & 6,3 & 8,7 & 17,5 & 10,3 & 21,8 & 26,2 & 9,1 & 3,84 & 1,95 & $-0,58$ & $-0,83$ \\
\hline AS7+(Q40) & 1,6 & 2,4 & 3,6 & 18,3 & 34,5 & 29,8 & 9,9 & 4,41 & 1,80 & $-1,45$ & 1,14 \\
\hline OV1-(Q8) & 2,0 & 6,7 & 10,7 & 21,4 & 27,8 & 25,8 & 5,6 & 4,27 & 1,64 & $-1,05$ & 0,54 \\
\hline OV2+(Q17) & 0,4 & 1,2 & 3,2 & 25,8 & 35,3 & 31,0 & 3,2 & 4,78 & 1,27 & $-1,81$ & 4,51 \\
\hline OV3-(Q20) & 1,2 & 6,7 & 12,7 & 13,1 & 31,3 & 19,8 & 15,1 & 3,81 & 2,00 & $-0,82$ & $-0,57$ \\
\hline OV4-(Q24) & 1,6 & 2,0 & 7,5 & 7,5 & 24,2 & 50,0 & 7,1 & 4,79 & 1,75 & $-1,65$ & 1,79 \\
\hline OV5+(Q25) & 0,0 & 2,0 & 1,6 & 19,8 & 33,7 & 34,9 & 7,9 & 4,66 & 1,63 & $-1,79$ & 2,70 \\
\hline OV6+(Q32) & 2,0 & 2,8 & 3,2 & 15,9 & 31,0 & 38,5 & 6,7 & 4,66 & 1,68 & $-1,62$ & 1,92 \\
\hline OV7-(Q35) & 2,0 & 1,6 & 5,6 & 7,5 & 20,6 & 57,9 & 4,8 & 5,03 & 1,59 & $-1,95$ & 3,12 \\
\hline
\end{tabular}

A Autonomia; DM Domínio do Meio; CP Crescimento Pessoal; RP Relações Positivas; AS Aceitação de Si; OV Objetivos de Vida

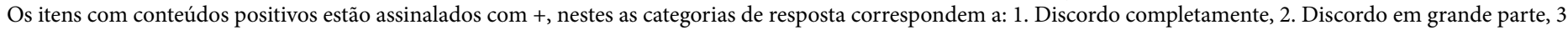
Discordo parcialmente, 4 . Concordo parcialmente, 5 . Concordo em grande parte e 6 . Concordo completamente

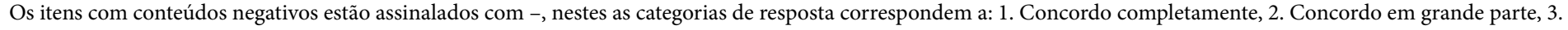

Concordo parcialmente, 4. Discordo parcialmente, 5. Discordo em grande parte e 6. Discordo completamente

* valor calculado sem a substituição dos valores omissos

As dimensões com pontuações médias mais baixas foram a Autonomia $(\mathrm{M}=30,90 ; \mathrm{DP}=5,07)$, o Domínio do Meio $(M=31,00 ; D P=4,90)$ e a Aceitação de Si $(\mathrm{M}=31,07 ; \mathrm{DP}=6,53)$, enquanto as que obtiveram valores mais elevados foram as dimensões Crescimento Pessoal $(M=35,71 ; D P=4,10)$, Objetivos de Vida $(M=34,45$; $\mathrm{DP}=4,64)$ e as Relações Positivas $(\mathrm{M}=33,95 ; \mathrm{DP}=4,64)$.

Para testar se o modelo teórico se adapta ao modelo empírico recorremos à AFC. Não se encontraram valores de assimetria $<$ a $|3|$ e de curtose $<$ a $|10|$, aceitando-se a normalidade multivariada. Testamos dois modelos diferentes, o modelo 1, com seis fatores oblíquos correlacionados e o modelo 2 , de seis fatores associados a um de ordem superior. Os resultados mostraram um ajustamento sofrível de ambos os modelos (tabela 2), todavia o primeiro mostrou melhores índices que o segundo, pelo que se optou por analisar os índices de modificação sugeridos para este modelo. A análise das estimativas dos parâmetros revelou que os fatores estavam altamente correlacionados e que todos os índices estandardizados saturaram no respetivo fator. O modelo 1 foi reespecificado com a correlação dos erros dos itens 1 e 4 do Domínio do Meio, tendo sido obtidos os índices de ajustamento correspondentes ao modelo 3 (tabela 2).
Como estes índices continuaram a mostrar um ajustamento sofrível, evoluiu-se com uma nova análise retirando dois itens com pesos fatoriais mais baixos (DM1 $=0,363$ e OV1=0,344) e cujos resíduos da matriz estandardizada de covariâncias mostraram valores acima de 2,58. Embora os índices de ajustamento tenham melhorado (modelo 4) estes continuam a mostrar valores pouco aceitáveis sobretudo de CFI. Ao consultar os índices de modificação, verificámos que se correlacionarmos os erros dos itens RP1 e RP5; DM2 e DM5; OV2 e OV6 o modelo melhorava, tendo sido efetuada nova análise (modelo 5).

Tabela 2 - Índices de Ajustamento aos Modelos Fatoriais Testados

\begin{tabular}{|l|l|l|l|l|l|l|}
\hline & X2/gl & RMR & GFI & CFI & $\begin{array}{l}\text { RM- } \\
\text { SEA }\end{array}$ & ECVI \\
\hline Modelo 1 & 1,840 & 0,179 & 0,782 & 0,794 & 0,058 & 6,688 \\
\hline Modelo 2 & 1,976 & 0,190 & 0,758 & 0,756 & 0,062 & 7,117 \\
\hline Modelo 3 & 2,159 & 0,091 & 0,729 & 0,821 & 0,064 & 7,704 \\
\hline Modelo 4 & 2,184 & 0,088 & 0,735 & 0,821 & 0,069 & 7,065 \\
\hline Modelo 5 & 2,051 & 0,085 & 0,750 & 0,833 & 0,065 & 6,680 \\
\hline
\end{tabular}

O modelo 5, adotado neste estudo encontra-se representado na Figura 1. 
Figura 1 - Estrutura do modelo final em análise

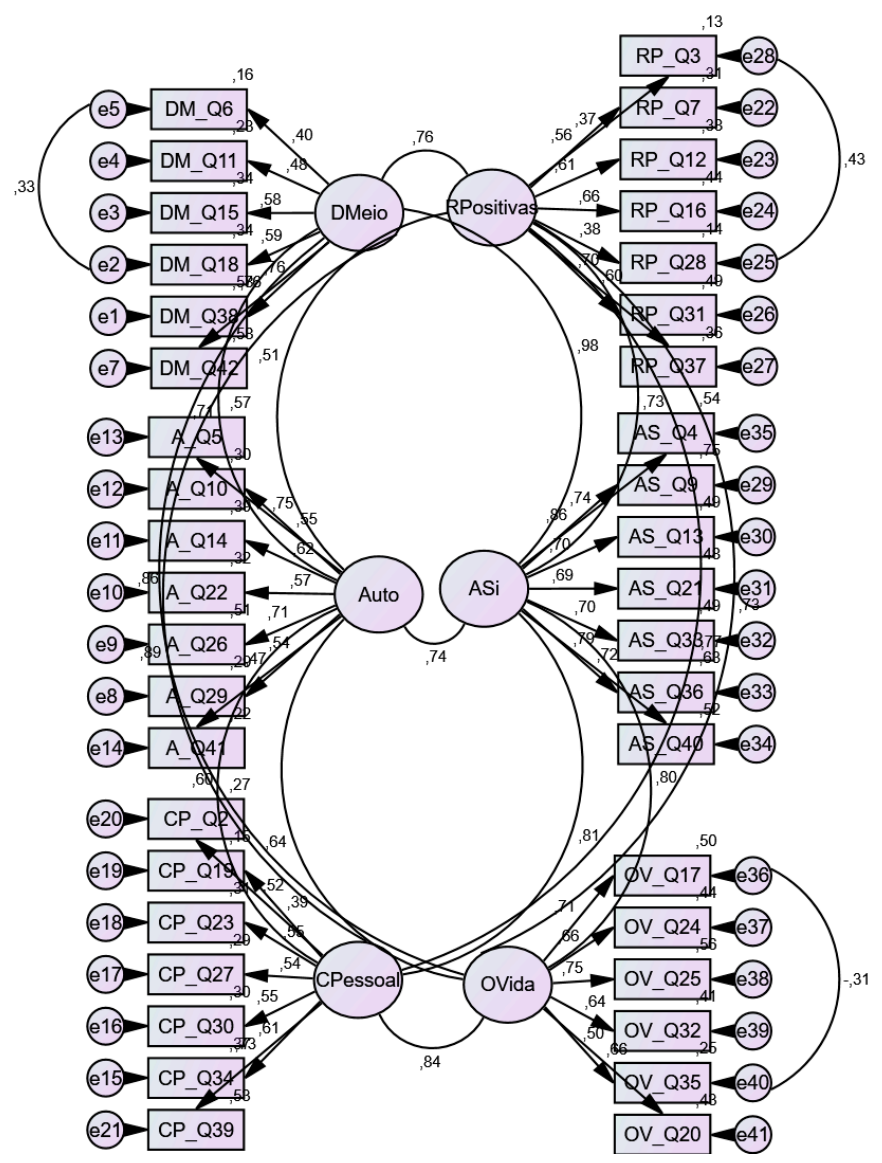

Procedemos à análise do coeficiente alfa de Cronbach para cada dimensão, tendo sido obtidos valores que variam entre 0,71 e 0,82 sugestivos de uma razoável a boa consistência interna (tabela 3 ). As correlações item escala total corrigidas mostram valores superiores a 0,34 , consideradas moderadas.

Tabela 3 - Valores de Correlação Item Escala Total e Valores do Coeficiente Alfa de Cronbach

\begin{tabular}{|l|l|l|l|l|l|l|}
\hline Item $^{*}$ & $\begin{array}{l}\text { Auto- } \\
\text { nomia }\end{array}$ & $\begin{array}{l}\text { Domínio } \\
\text { meio }\end{array}$ & $\begin{array}{l}\text { Cresci- } \\
\text { mento } \\
\text { pessoal }\end{array}$ & $\begin{array}{l}\text { Relações } \\
\text { positivas }\end{array}$ & $\begin{array}{l}\text { Aceita- } \\
\text { ção de } \\
\text { Si }\end{array}$ & $\begin{array}{l}\text { Objeti- } \\
\text { vos de } \\
\text { vida }\end{array}$ \\
\hline Item 1 & 0,44 & & 0,39 & 0,45 & 0,55 & \\
\hline Item 2 & 0,44 & 0,43 & 0,39 & 0,47 & 0,63 & 0,60 \\
\hline Item 3 & 0,46 & 0,35 & 0,41 & 0,42 & 0,45 & 0,58 \\
\hline Item 4 & 0,44 & 0,47 & 0,51 & 0,55 & 0,54 & 0,60 \\
\hline Item 5 & 0,54 & 0,62 & 0,39 & 0,34 & 0,60 & 0,65 \\
\hline Item 6 & 0,39 & 0,71 & 0,42 & 0,54 & 0,59 & 0,55 \\
\hline Item 7 & 0,34 & 0,57 & 0,50 & 0,44 & 0,57 & 0,39 \\
\hline $\begin{array}{l}\text { a Cron- } \\
\text { bach }\end{array}$ & 0,72 & 0,77 & 0,71 & 0,74 & 0,82 & 0,80 \\
\hline
\end{tabular}

* a numeração apresentada somente assegura a ordem em que cada item aparece na escala global.

As dimensões da EBEP apresentam correlações moderadas a fortes que variam entre 0,31 e 0,73 (tabela 4).
Todas as correlações mostram valores significativos ao nível de 0,01. Os valores encontrados sugerem que as dimensões da EBEP estão relacionadas sem, contudo, haver redundância das medidas.

Tabela 4 - Matriz de Correlação das Dimensões da EBEP

\begin{tabular}{|l|l|l|l|l|l|l|}
\hline $\begin{array}{l}\text { Subes- } \\
\text { cala }\end{array}$ & A & DM & CP & RP & AS & OV \\
\hline A & - & $0,55^{\star *}$ & $0,45^{\star *}$ & $0,31^{\star *}$ & $0,58^{\star *}$ & $0,45^{\star *}$ \\
\hline DM & & - & $0,60^{\star *}$ & $0,52^{\star *}$ & $0,73^{\star *}$ & $0,63^{\star *}$ \\
\hline P & & & - & $0,55^{\star *}$ & $0,61^{\star *}$ & $0,66^{\star *}$ \\
\hline RP & & & & - & $0,52^{\star *}$ & $0,53^{\star *}$ \\
\hline AS & & & & & - & $0,62^{\star *}$ \\
\hline $\begin{array}{l}\text { Média } \\
(\mathrm{DP})\end{array}$ & 30,90 & 31,00 & 35,71 & 33,95 & 31.70 & 34,45 \\
\cline { 2 - 7 } & $(5,07)$ & $(4,90)$ & $(4,10)$ & $(4,64)$ & $(6,53)$ & $(4,64)$ \\
\hline
\end{tabular}

A Autonomia; DM Domínio do Meio; CP Crescimento Pessoal; RP Relações Positivas; AS Aceitação de Si; OV Objetivos de Vida ${ }^{* *} \mathrm{p}<0,001$

Correlacionando as dimensões da EBEP com as do SF36, apenas se encontrou uma correlação estatisticamente significativa entre a Autonomia e a Saúde Mental $(r=0,137 ; p=0,03)$. Não se registaram diferenças significativas na perceção da EBEP entre homens e mulheres, exceto na dimensão Domínio do Meio onde os homens apresentam valores mais elevados $(\mathrm{M}=30,69 ; \mathrm{DP}=6,36)$ do que as mulheres $(M=27,85 ; D P=7,02)$, sendo estas diferenças estatisticamente significativas $(t(250)=2,56$; $\mathrm{p}=0,011$ ).

Verificou-se uma correlação positiva fraca, mas significativa, entre a idade e a maioria das dimensões da EBEP. Em relação à escolaridade apenas se encontrou uma relação fraca entre esta e a Autonomia e entre a escolaridade e a Aceitação de Si (Tabela 5).

Tabela 5 - Correlação entre as Dimensões da EBEP e as Variáveis Sociodemográficas Idade e Escolaridade

\begin{tabular}{|c|c|c|c|c|c|c|}
\hline $\begin{array}{l}\text { Subes- } \\
\text { cala }\end{array}$ & $\begin{array}{l}\text { Auto- } \\
\text { nomia }\end{array}$ & $\begin{array}{l}\text { Domínio } \\
\text { meio }\end{array}$ & $\begin{array}{l}\text { Cresci- } \\
\text { mento } \\
\text { pessoal }\end{array}$ & $\begin{array}{l}\text { Relações } \\
\text { positivas }\end{array}$ & $\begin{array}{l}\text { Aceita- } \\
\text { ção de } \\
\text { Si }\end{array}$ & $\begin{array}{l}\text { Objeti- } \\
\text { vos de } \\
\text { vida }\end{array}$ \\
\hline Idade & $0,34^{* *}$ & $0,23^{* *}$ & $0,13^{*}$ & 0,11 & $0,24^{\star *}$ & 0,10 \\
\hline $\begin{array}{l}\text { Esco- } \\
\text { laridade }\end{array}$ & $\begin{array}{l}0,15^{\star} \\
<0,01\end{array}$ & 0,12 & 0,09 & 0,12 & $0,15^{\star *}$ & 0,06 \\
\hline
\end{tabular}

\section{DISCUSSÃO}

Analisando o conteúdo das afirmações que reuniram maior percentagem de respostas omissas, constatamos que se reportam a aspetos nem sempre fáceis de responder por pessoas jovens (maioria da nossa amostra) com uma experiência de vida ainda limitada (p.e. "consegui construir um lar e um estilo de vida que é muito do meu agrado" e "sinto que muitas das pessoas que 
conheço conseguiram tirar mais partido da vida do que eu"), ou porque os itens poderão integrar termos cujo significado desconhecem ("as minhas atividades diárias parecem-me frequentemente triviais e pouco importantes"), ou ainda serem muito gerais e difíceis de particularizar ("tenho tendência a ser influenciado por pessoas com opiniões firmes").

De forma global, a tendência de resposta no presente estudo situou-se acima do ponto médio, expressando que os participantes tendem a ter uma visão positiva acerca do seu bem-estar psicológico. Nos itens com conteúdo positivo as respostas aos níveis mais baixos de bem-estar incluíram uma percentagem baixa de respostas, fixando-se entre 1 a $3 \%$, na sua maioria. Esta mesma tendência de resposta foi encontrada nos estudos portugueses (Novo, 2003), bem como nos estudos com a versão de 42 itens (Abbott et al., 2010; Boylan \& Ryff, 2015).

As dimensões Crescimento Pessoal e Objetivos de Vida, valorização de aspetos da afirmação pessoal, do individualismo e da independência, foram as que apresentaram valores mais elevados e as que apresentaram médias mais baixas foram as dimensões Autonomia e Domínio do Meio, sendo estes resultados concordantes com outros trabalhos de Ryff (Ryff et al., 1995; Boylan \& Ryff, 2015) e com estudos realizados em Portugal (Novo, 2003, 2005).

As correlações item escala total corrigidas mostram valores superiores a 0,34 , consideradas moderadas, resultados sobreponíveis a Novo (2003). Os valores do coeficiente alfa de Cronbach encontrados variaram entre 0,71 (na dimensão Crescimento Pessoal) a 0,82 (na dimensão Aceitação de $\mathrm{Si}$ ), sugerindo valores de uma razoável a boa consistência interna. O estudo de Boylan e Ryff (2015), com a mesma estrutura da EBEP em estudo, mostrou valores de consistência interna sobreponíveis.

Através da AFC o modelo final adotado mostrou índices sofríveis. Parece haver alguma consistência nos resultados da AFC desta escala, mostrando índices de ajustamento, nomeadamente valores de CFI abaixo dos valores considerados aceitáveis (Chen, Jing, Hayes \& Lee, 2013; Fernandes et al., 2010; Gao \& McLellan, 2018; van Dierendonck, 2005).

Verificamos que a idade se correlacionou de forma fraca, mas positiva e com significância estatística com a Autonomia, Domínio do Meio, Crescimento Pessoal e Aceitação de Si, sugerindo que os participantes experienciam melhor bem-estar psicológico nestes domínios, conforme vão avançando na idade.
Também Boylan e Ryff (2015) encontraram associações entre a idade e estas dimensões, mas com valores de correlação muito baixas (entre 0,06 e 0,24), bem como fraca significância estatística $(\mathrm{p} \leq .05)$. Se estes domínios de bem-estar englobam aspetos como o autoconhecimento e uma atitude positiva em relação a si, a melhoria da autonomia, o controlo sobre o ambiente, bem como a possibilidade de crescimento pessoal, parece provável que a perceção de bem-estar nestas dimensões sofra alterações ao longo do ciclo de vida da pessoa. A nossa amostra contempla adultos jovens que se encontram numa fase de grande desenvolvimento pessoal, social e profissional, o que pode explicar a evolução positiva nestes domínios. Estes resultados podem sugerir que a autonomia, o domínio ambiental, as relações positivas com os outros e os reflexos de autoaceitação sejam domínios distintos do funcionamento psicológico positivo. Springer, Pudrovska e Hausen (2011) exploraram a variação da idade na EBEP em dois estudos e verificaram que as variações nas diferentes dimensões foram excecionalmente pequenas, encontrando inclusive inconsistências.

No entanto, a amplitude da idade nestes estudos variou entre 32 e os 75 anos, diferindo da nossa que engloba adultos jovens e não inclui pessoas acima dos 68 anos. Também, estudos transversais e longitudinais efetuados sugerem que objetivos na vida, crescimento pessoal e outras dimensões existenciais de bem-estar tendem a diminuir desde a idade adulta média até a idade avançada (Ryff, 2014; Springer et al., 2011).

Verificamos uma relação fraca, positiva e estatisticamente significativa entre a escolaridade e a Autonomia e a escolaridade e a Aceitação de Si. Boylan e Ryff (2015) também encontraram valores positivos de correlação, embora muito fracos, entre esta variável e as duas dimensões atrás descritas.

A educação permite que a pessoa desenvolva um conjunto de competências que promove a capacidade de reflexão e a tomada de decisão autónoma, podendo assim tornar-se menos influenciável e mais resistente a pressões externas. Ajuda também a que, através de processos introspetivos, a pessoa possa desenvolver uma atitude mais positiva em relação a si, reconhecendo e aceitando as suas qualidades e defeitos. Vários estudos têm vindo a demonstrar uma associação positiva entre as várias dimensões da EBEP e o nível educacional, até porque a educação frequentemente é acompanhada por melhorias nas condições económicas e sociais (Novo, 2003; Novo, 2005) 
Encontramos ainda a inexistência de diferenças significativas na perceção da EBEP entre homens e mulheres, exceto na dimensão Domínio do Meio, onde os homens apresentam valores mais elevados do que as mulheres. Uma possível explicação para os nossos resultados poderá estar relacionada com aspetos culturais ainda enraizados na sociedade portuguesa, onde a mulher poderá ter que despender um maior esforço no sentido de desenvolver uma participação ativa bem como ganhar mais domínio do ambiente envolvente. $O$ facto de alguns estudos com a EBEP mostrarem diferenças entre géneros poderão estar relacionados com desigualdades a nível sociocultural na gestão de direitos e oportunidades de desenvolvimento entre países ( $\mathrm{Li}, \mathrm{Kao}, \& \mathrm{Wu}$, 2015).

\section{CONCLUSÕES}

A EBEP tem sido utilizada em diferentes contextos e culturas mostrando a sua evidente adequação para avaliar o bem-estar psicológico. A versão reduzida em estudo mostrou que, se eliminássemos dois itens, a escala estaria melhor adaptada a uma amostra maioritariamente jovem.

A EBEP mostra ser discriminativa para a idade, género, escolaridade e condição de saúde mental. O estudo da consistência interna mostrou que todas as dimensões apresentam valores psicométricos aceitáveis. Os resultados da AFC vêm realçar que a estrutura fatorial da escala não mostra ser tão clara quanto o quadro teórico sugerido, requerendo novas investigações.

\section{IMPLICAÇÕES PARA A PRÁTICA CLÍNICA}

O bem-estar psicológico é um indicador emocional relevante com interesse clínico. As competências emocionais são cada vez mais alvo de atenção dos enfermeiros e de outros técnicos de saúde, na medida em que facilitam uma melhor adaptação e aceitação do estado e condição de saúde da pessoa.

A aplicação deste instrumento permite assim uma melhor avaliação e monitorização deste construto. A EBEP é um instrumento que tem sido utilizado em muitos contextos culturais o que manifesta a sua boa aceitação e validade clínica.

\section{REFERÊNCIAS BIBLIOGRÁFICAS}

Abbott, R., Ploubidis, G., Huppert, F., Kuh, D. \& Croudace, T. (2010). An evaluation of the precision of measurement of Ryff's Psychological Well-Being Scales in a population sample. Social Indicators Research, 97(3), 357-373. doi. 10.1007/s11205-009-9506-x

Boylan, J.M. \& Ryff, C.D. (2015). Psychological wellbeing and metabolic syndrome: findings from the midlife in the United States national sample. Psychosomatic medicine, 77(5), 548-58. doi:10.1097/ PSY.0000000000000192

Chen, F.F., Jing, Y., Hayes, A. \& Lee, J.M. (2013). Two concepts or two approaches? A bifactor analysis of psychological and subjective well-being. Journal of Happiness Studies, 14(3), 1033-1068. doi: 10.1007/s10902012-9367-x

Fernandes, H., Vasconcelos-Raposo, J., Bertelli, R. \& Almeida, L. (2011). Satisfação escolar e bem-estar psicológico em adolescentes portugueses. Revista Lusófona de Educação, 18, 155-172. Disponível em: http:// www.scielo.mec.pt/scielo.php?script=sci_arttext\&pid $=$ S1645-72502011000200011

Fernandes, H., Vasconcelos-Raposo, J. \& Teixeira, C. (2010). Preliminary Analysis of the Psychometric Properties of Ryff's S Scales of Psychological Well-Being in Portuguese Adolescents. The Spanish Journal of Psychology, 13(2), 1032-43. Doi: 10.1017/S1138741600002675

Ferreira P.L. (2000). Criação da versão portuguesa do MOS SF-36. Parte II - Testes de validação. Acta Médica Portuguesa,13 (3),119-27. Disponível em: https://actamedicaportuguesa.com/revista/index.php/amp/index

Gao, J. \& McLellan, R. (2018). Using Ryff's scales of psychological well-being in adolescents in mainland China. BMC Psychology, 6(17). Doi: 10.1186/s40359018-0231-6

Kline, R.B. (2010). Principles and practice of structural equation modelling. (3rd ed). New York: Guilford. 
Li, R.H., Kao, C.M. \& Wu, Y.Y. (2015). Gender differences in psychological well-being: tests of factorial invariance. Quality Life Research, 24(11), 2577-81. Doi. 10.1007/s11136-015-0999-2

Malla, S. S. (2013). Cross-cultural Validity of Ryff's Well-being Scale in India. Asia-Pacific Journal of Management Research and Innovation, 9(4), 379-387. Doi.10.1177/2319510x14523107

Morozink, J.A., Friedman, E.M., Coe, C.L. \& Ryff, C.D. (2010). Socioeconomic and psychosocial predictors of interleukin-6 in the MIDUS national sample. Health Psychology, 29, 626-635. Doi. 10.1037/a0021360

Novo, R. F. (2003). Para Além da Eudaimonia - O BemEstar Psicológico em Mulheres de Idade Adulta avançada. Lisboa: Fundação Calouste Gulbenkian e Fundação para a Ciência e Tecnologia.

Novo, R. F. (2005). We need more than self-reports: contributo para a reflexão sobre as estratégias de avaliação do bem-estar. Revista de Psicologia, Educação e Cultura, 9, 477-495. Disponível em: http://repositorio. ul.pt/bitstream/10451/17832/1/2005\%20BEP\%20Revista\%20Psicologia\%20Educa\%C3\%A7\%C3\%A3o\%20 $\% 26 \% 20$ Cultura.pdf

Ryff, C. D. (1989). Happiness is everything, or is it? Explorations on the meaning of psychological well-being. Journal of Personality and Social Psychology, 57(6), 1069-1081. Doi: 10.1037/0022-3514.57.6.1069
Ryff, C.D. (2014). Psychological well-being revisited: Advances in the science and practice of eudaimonia. Psychotherapy and Psychosomatics, 83(1), 10-28. Doi.10.1159/000353263

Ryff, C. D. \& Keyes, C. L. (1995). The Structure of Psychological Well-Being Revisited. Journal of Personality \& Social Psychology, 69, 719-727. Doi: 10.1037/00223514.69.4.719

Ryff, C. D. \& Singer, B. H. (2006). Best news yet on the six-factor model of well-being. Social Science Research, 35(4), 1103-1119. Doi: 10.1016/j.ssresearch.2006.01.002

Springer, K.W., Pudrovska, T. \& Hauser, R.M. (2011). Does psychological well-being change with age?: Ryff's model of psychological well-being. Social Science Research, 40, 392-398. Doi:10.1016/j.ssresearch.2010.05.008

van Dierendonck, D. (2005). The construct validity of Ryff's scales of psychological well-being and it's extension with spiritual well-being. Personality and Individual Differences, 36, 629-643. Doi: 10.1016/S01918869(03)00122-3

Villarosa, J. \& Ganotice, F. (2018). Construct Validation of Ryff's Psychological Well-being Scale: Evidence from Filipino Teachers in the Philippines. Philippine Journal of Psychology, 51(1), 1-20. 